\title{
Signal to Noise Ratio
}

National Cancer Institute

\section{Source}

National Cancer Institute. Signal to Noise Ratio. NCI Thesaurus. Code C94983.

The ratio of a measure of useful information from a system to that of a measure of interfering unwanted, often random, information that is also present. 